\title{
Breast cancer in neurofibromatosis 1: survival and risk of contralateral breast cancer in a five country cohort study
}

\author{
D. Gareth R. Evans, MD ${ }^{1,2,3,4}$, Roope A. Kallionpää, MSc (pharm) ${ }^{5}{ }^{5}$, Maurizio Clementi, MD $^{6}$, \\ Eva Trevisson, $\mathrm{MD}{ }^{6}{ }^{6}$, Victor-Felix Mautner, MD ${ }^{7}$, Sacha J. Howell, PhD ${ }^{1,4,8}$, Lauren Lewis, BSc $^{2}$, \\ Ouidad Zehou, $\mathrm{MD}^{9}$, Sirkku Peltonen, $\mathrm{MD} \mathbb{1 0}^{10}$, Antonella Brunello, MD ${ }^{11}{ }^{11}$, \\ Elaine F. Harkness, PhD ${ }^{4,12}$, Pierre Wolkenstein, $\mathrm{MD}^{9}$ and Juha Peltonen, $\mathrm{MD} \mathbb{D}^{5}$
}

Purpose: Neurofibromatosis 1 (NF1) is an autosomal dominant condition caused by pathogenic variants of the NF1 gene. A markedly increased risk of breast cancer is associated with NF1. We have determined the breast cancer survival and risk of contralateral breast cancer in NF1.

Methods: We included 142 women with NF1 and breast cancer from five cohorts in Europe and 335 women without NF1 screened for other familial breast cancers. Risk of contralateral breast cancer and death were assessed by Kaplan-Meier analysis with delayed entry.

Results: One hundred forty-two women with NF1 were diagnosed for breast cancer at a median age of 46.9 years (range 27.0-84.3 years) and then followed up for 1235 person-years (mean $=8.70$ years). Twelve women had contralateral breast cancer with a rate of 10.5 per 1000 years. Cumulative risk for contralateral breast cancer was $26.5 \%$ in 20 years. Five and 10 -year all-cause survival was
$64.9 \%(95 \%$ confidence interval $[\mathrm{CI}]=54.8-76.8)$ and $49.8 \%(95 \%$ $\mathrm{CI}=39.3-63.0)$. Breast cancer-specific 10 -year survival was $64.2 \%$ $(95 \% \quad \mathrm{CI}=53.5-77.0 \%) \quad$ compared with $91.2 \% \quad(95 \% \quad \mathrm{CI}=$ 87.3-95.2\%) in the non-NF1 age-matched population at increased risk of breast cancer.

Conclusion: Women with NF1 have a substantial contralateral breast cancer incidence and poor survival. Early start of breast cancer screening may be a way to improve the survival.

Genetics in Medicine (2020) 22:398-406; https://doi.org/10.1038/s41436019-0651-6

Keywords: breast cancer; neurofibromatosis 1; NF1; prognosis; mammography screening

\section{INTRODUCTION}

Neurofibromatosis 1 (NF1; OMIM 162200), is an autosomal dominant tumor predisposition syndrome with a birth incidence of 1 in 1900-3000 and prevalence of around 1 in 2000-4000. ${ }^{1,2}$ NF1 demonstrates complete penetrance for the characteristic lesions but significant variability in clinical phenotype due to differences in the site and type of genetic defect in the NF1 gene and additional genetic and environmental factors that are not well determined. ${ }^{3}$ Clinical features in individuals can be difficult to predict, even within families, which makes genetic counseling imprecise. Approximately $50 \%$ of cases are familial but the remaining $50 \%$ are sporadic and due to de novo aberrations of the NF1 gene. ${ }^{4}$

National Institutes of Health (NIH) diagnostic criteria for NF1 require two or more of the following: café-au-lait spots, neurofibromas ( 2 or more), skin fold freckling, Lisch nodules, optic glioma, osseous lesions, or a family history of the condition in a first-degree relative. Genetic testing of the NF1 gene has also allowed molecular diagnosis when the syndrome is suspected: DNA analysis coupled with RNA sequencing has high sensitivity of around $96 \%$ in both de novo and inherited NF1. ${ }^{5}$ An associated malignancy risk has long been recorded for malignant peripheral nerve sheath tumors (MPNSTs) in

${ }^{1}$ The Christie NHS Foundation Trust, Manchester, UK; ${ }^{2}$ Manchester Centre for Genomic Medicine, Central Manchester University Hospitals NHS Foundation Trust, Manchester, UK; ${ }^{3}$ Division of Evolution and Genomic Sciences, School of Biological Sciences, Faculty of Biology, Medicine and Health, University of Manchester, Manchester Academic Health Science Centre, Manchester, UK; ${ }^{4}$ Genesis Breast Cancer Prevention Centre and Nightingale Breast Screening Centre, University Hospital of South Manchester, Manchester Academic Health Science Centre, Manchester, UK; ${ }^{5}$ Institute of Biomedicine, University of Turku, Turku, Finland; ${ }^{6}$ Clinical Genetics Unit, Deparment of Women's and Children's Health, University of Padova, Padova, Italy: ${ }^{7}$ Phacomatoses Department, Department of Neurology, University Medical Centre Hamburg-Eppendorf, Hamburg, Germany: ${ }^{8}$ Division of Cancer Sciences, Faculty of Biology, Medicine and Health, The University of Manchester, Manchester Academic Health Science Centre, Manchester, UK; ${ }^{9}$ Referral Centre for Neurofibromatoses Dermatology, Hôpital Henri Mondor, APHP, UPEC, Créteil, France; ${ }^{10}$ Department of Dermatology, University of Turku and Turku University Hospital, Turku, Finland; ${ }^{11}$ Medical Oncology 1 Unit, Department of Oncology, Istituto Oncologico Veneto IOV - IRCCS, Padova, Italy; ${ }^{12}$ Division of Informatics, Imaging and Data Sciences, Faculty of Biology, Medicine and Health, University of Manchester, Manchester, UK. Correspondence: Juha Peltonen (juhpel@utu.fi)

These authors contributed equally: D. Gareth R. Evans, Roope A. Kallionpää. 
particular. ${ }^{6}$ Central nervous system tumors usually present earlier in childhood and mostly comprise of low-grade pilocytic astrocytomas of the optic radiations or brainstem. In addition to these tumors, patients with NF1 are at a higher risk of gastrointestinal stromal tumors (GISTs), rhabdomyosarcomas, and pheochromocytoma. ${ }^{7}$ The link with an increased incidence of breast cancer has been debated for the last 10-15 years but more conclusive data have now confirmed this link. ${ }^{8-13}$

The definitive study came from Finland where populationlevel data were taken from all secondary and tertiary medical centers covering the whole of the 5.4 million Finnish population from 1987 to 2011 . $^{72}$ Seven hundred thirty-seven women with NF1 were identified and verified according to NIH criteria. Carcinoma-specific survival of patients with NF1 was compared with that of matched controls from the Finnish Cancer Registry. Overall the lifetime risk of any cancer was $59.6 \%$ in NF1 compared with $30.8 \%$ in the general Finnish population. The standardized incidence ratio (SIR) for breast cancer was 3.04 (95\% confidence interval [CI] 2.06 to $4.31 ; P<0.001$ ) overall and 11.1 (95\% CI 5.56 to $19.5 ; P<0.001)$ in women $<40$. Both overall mortality (standardized mortality ratio [SMR] 7.23; 95\% CI 5.58 to $9.19 ; P<0.001)$ and breast cancer-specific mortality (SMR 5.20; 95\% CI 2.38 to 9.88; $P<0.001$ ) were increased among women with NF1 versus controls.

With the identification of the NF1 gene as a driver in breast cancer in the Cancer Genome Atlas (TCGA) ${ }^{14}$ we went on to analyze TCGA data and, as with Suarez-Cabrera ${ }^{15}$ and Wallace et al., ${ }^{16}$ demonstrated NF1 aberrations in 33\% of breast cancers with a significant enrichment in estrogen receptor (ER) negative and HER2 positive subtypes. ${ }^{12}$

In the majority of genetic predisposition syndromes such as defects in BRCA1, BRCA2, TP53, CHEK2, and ATM the rate of contralateral breast cancer is increased. A number of case reports have recorded the presence of bilateral breast cancer in NF1. ${ }^{9,10,12}$ However, no formal quantification of this risk has been published. We have combined five large series of NF1 cases and assessed the risks of contralateral breast cancer in five European countries, as well as survival after breast cancer diagnosis.

\section{MATERIALS AND METHODS}

All patients with confirmed NF1 by NIH criteria who had developed breast cancer were ascertained from the Finnish population-based NF registry, ${ }^{2}$ the Manchester regional NF1 registry (UK), ${ }^{6,9}$ the Paris NF1 registry (France), ${ }^{17}$ Hamburg neurofibromatosis clinic (Germany), ${ }^{18}$ and the Padova NF1 clinic (Italy). ${ }^{19}$ The collection of the study cohorts was approved by Ethics Committee of the Hospital District of Southwest Finland, Central Manchester Research Ethics Committee, institutional review board CPP Ile-de-France IV, and Ethics Committee Board in Hamburg. Informed consent was obtained unless exempt based on the use of retrospective register data. The patients were mainly diagnosed with NF1 based on clinical symptoms as genetic testing has become widely available only recently. The Manchester and Finnish populations represent close to, or complete, ascertainment of patients with NF1 in defined regions in North West England (population 5 million) and all Finland (population 5 million). The other clinics are specialized NF1 clinics nonetheless with reasonably high ascertainment of patients with NF1. Total patient numbers in each cohort were Finland $=1476$ (770 female); Manchester $=$ 2148 (1099 female); Paris = 1895 (1011 female), Hamburg = 2019 (1011 female), Padova $=811$ (464 female) (Fig. 1). Breast cancer diagnoses were confirmed from patient notes, histology

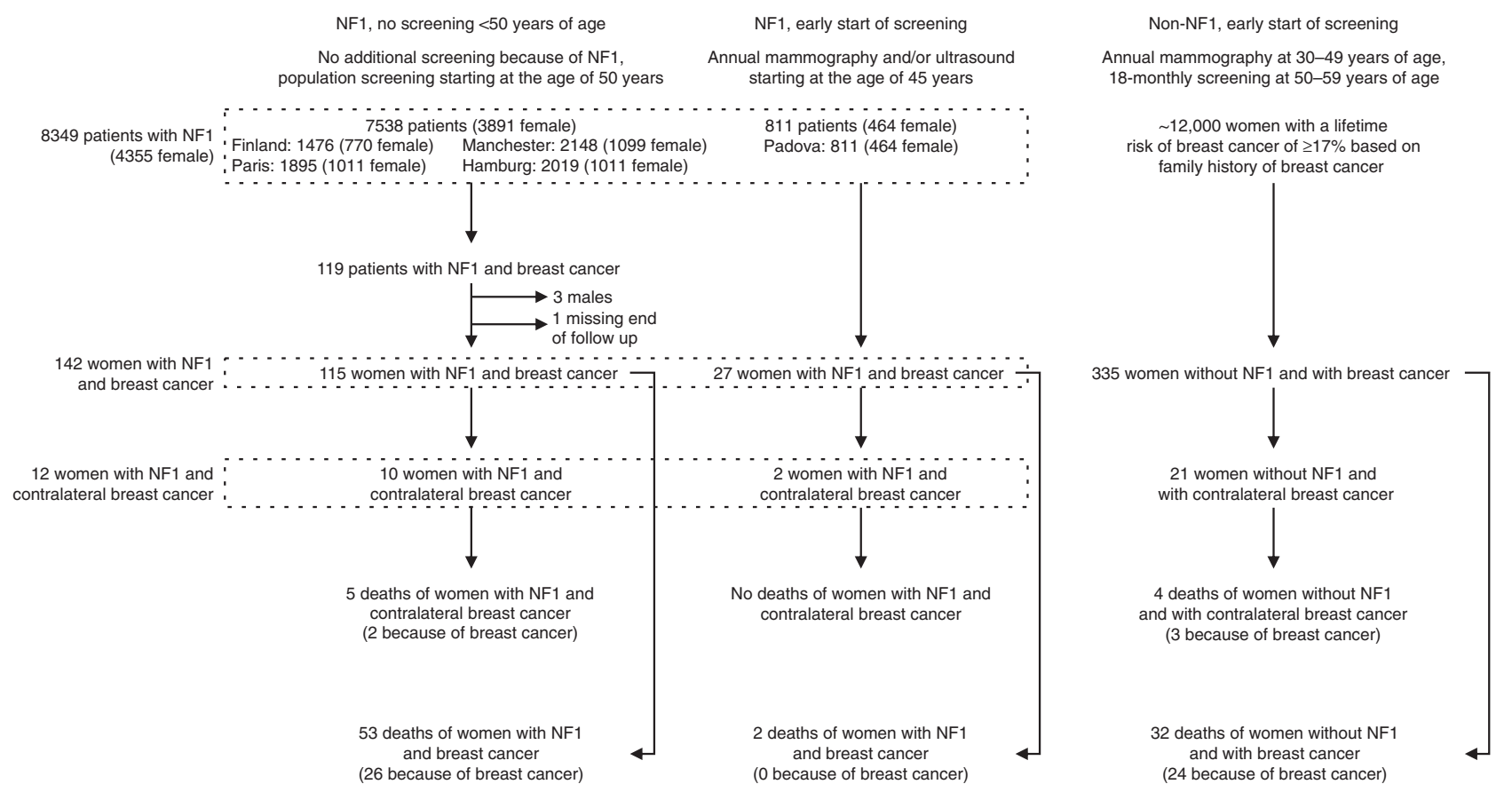

Fig. 1 Patients included in the study. NF1 neurofibromatosis 1. 
reports, and cancer registry notifications. Data of last follow-up were based on the date of death, last clinical contact, or information from national registries. Finnish data were censored at 31 December 2014.

Three groups of patients with breast cancer were analyzed: (1) patients with NF1 and no screening $<50$ years of age, (2) patients with NF1 and early start of screening for breast cancer, and (3) patients without NF1 and with early start of screening for breast cancer because of family history. Patients with NF1 and no additional screening came from Finland, Manchester, Paris, and Hamburg. They may have participated the general population mammography screening programs, starting at the age of 50 years and initiated 1989-2005 depending on the country, but they had no additional screening because of their NF1. Those in the four cohorts with no additional breast cancer screening were compared with the Padova clinic in which screening with annual mammography and/or ultrasound has been performed on all women with NF1 starting at the age of 45 years since 2009-2010.

Moreover, a non-NF1 cohort of 335 women who developed breast cancer while undergoing annual mammography screening aged 30-49 years and 18-monthly screening aged 50-59 years for familial breast cancer risk in Manchester was used to demonstrate prognosis in screened non-NF1 population. ${ }^{20}$ This family history clinic population was identified from 12,000 women screened due to a lifetime risk of breast cancer of $\geq 17 \%$ based on family history of breast cancer. Genetic testing typically occurred after inclusion in the cohort, thus avoiding bias toward certain variants. In addition to screen-detected breast cancers, all interval cancers occurring within 18 months of a normal mammogram were included. ${ }^{20}$ There were no women with NF1 and breast cancer in this cohort. Since the non-NF1 cohort consists of women with familial breast cancer risk and early start of screening, it is not intended to serve as a control for the effect of NF1 but rather to represent what can be achieved with screening.

In the survival analyses, death was considered as an event, and the follow-up was censored at emigration or last information from either registries or clinical contact. In the calculation of the risk for contralateral breast cancer, contralateral breast cancer was an event, and the follow-up was censored at death, emigration, or last information on patient status. The follow-up started at breast cancer diagnosis or, in a second analysis to allow for potential survival bias in those ascertained with NF1 after breast cancer, at the latter of breast cancer or entry to NF1 cohort (delayed entry). Cumulative risk was computed with and without the competing risk of death. Kaplan-Meier estimates of survival and risk were computed. The analyses were stratified by country and tumor grade. The statistical comparisons were based on Cox proportional hazards model and two-sided tests. The proportional hazards assumption was met. Fisher's exact test was used in comparisons of tumor characteristics. The R software version 3.3.0 (www.r-project.org) and package survival version $2.41-3$ were used for the statistical analyses.

\section{RESULTS}

One hundred forty-six patients with NF1 and breast cancer were identified from the five cohorts (Finland $=48$, Manchester $=35$, Padova $=27$, Paris $=20$, Hamburg $=16$; Fig. 1). Three cases of male breast cancer aged 40.7, 69.0, and 71.5 were not analyzed further. One female patient with NF1 and breast cancer was excluded because of lack of information on the end of follow-up. Thus, there were 142 women with NF1 who had their first breast cancer diagnosed at a median age of 46.9 years (range 27.0-84.3 years). There were 1235 years of clinical follow-up from breast cancer diagnosis to death, emigration, or last information on patient status among women with NF1 (mean $=8.70$ years, standard deviation $7.60)$. In the non-NF1 group, there were 2437 years of followup from 335 patients (mean 7.27, standard deviation 6.89).

Twelve women with NF1 had developed contralateral breast cancer at a median age of 53 years (range 28.5-62.8) with a total 1141 years of follow-up from the first breast cancer to the second breast cancer, death, emigration, or last information on patient status in the whole cohort. This equates to a rate of contralateral breast cancer of 10.5 per 1000 years, comparable with the screened family history clinic patients without NF1 (Fig. 2). Cumulative risk was 26.5\% (95\% CI $12.4-56.9)$ by 20 years in those who were alive and $15.7 \%$ (95\% CI 8.1-30.4) allowing for competing mortality. The first tumors in those diagnosed later with a second breast cancer were stage 1 as often as tumors in all patients of the five NF1 cohorts. While the first cancer was most often detected due to symptoms, the contralateral tumors were diagnosed in screening in half of the cases where information was available.

Of the 115 women with NF1 from the Finland, Manchester, Hamburg, and Paris cohorts without screening $<50$ years of age, $53(46.1 \%)$ had died $(0.28-30.6$ years post-breast cancer diagnosis), 26 from breast cancer (2/10 of the contralateral cases), 8 from other malignancies, 4 from cardiovascular disease, and 11 from other causes. Four died from unknown causes. Twenty-nine deaths $(25.2 \%$ of all cases, $54.7 \%$ of all deaths) occurred within 5 years, at least 20 from breast cancer (3 unknown). Overall 74/115 women had been diagnosed with breast cancer after inclusion into one of the four NF1 cohorts with 41 being identified as having NF1 a median of 5.1 years afterward (range 0.1-23.2 years).

Five and 10-year all-cause survival adjusting for those ascertained with NF1 after breast cancer diagnosis (delayed entry) was $64.9 \%$ (95\% CI 54.8-76.8) and $49.8 \%$ (95\% CI 39.3-63.0) among the unscreened patients with NF1. Using breast cancer-specific survival the 10-year survival was $64.2 \%$ (95\% CI 53.5-77.0\%) and for the screened non-NF1 familial population $91.2 \% \quad$ (95\% CI $87.3-95.2 \%)$. Kaplan-Meier analysis is shown in Fig. 2 with both the unadjusted and delayed entry curves provided. Tables 1 and 2 show that the more highly ascertained Manchester and Finnish populations showed poorer all-cause and breast cancer-specific survival. The screened cases from Padova had significantly better prognosis than the other four NF1 cohorts combined with hazard ratio $(\mathrm{HR})$ of $0.18(95 \% \mathrm{CI} 0.04-0.72 ; P=0.016)$. Most 

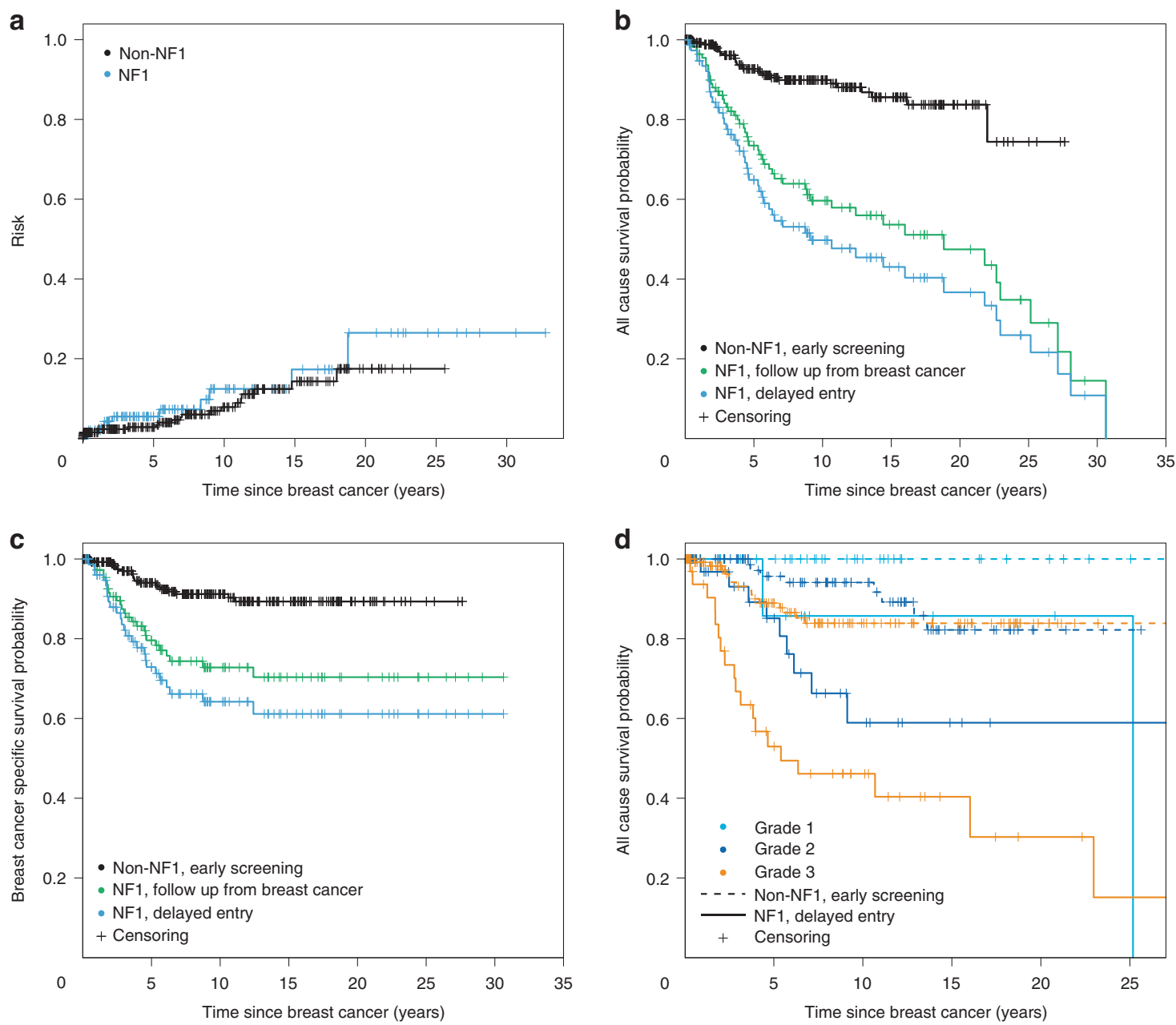

Fig. 2 Breast cancer survival and risk of contralateral breast cancer. a Contralateral breast cancer incidence after the first breast cancer in female patients with neurofibromatosis 1 (NF1) compared with screened family history clinic patients without NF1. b All-cause survival in unscreened patients with NF1 compared with screened family history clinic patients without NF1. The numbers of patients at risk at different time points are shown in Table 1. c Breast cancer-specific survival in unscreened patients with NF1 compared with screened family history clinic patients without NF1. The numbers of patients at risk at different time points are shown in Table 2. d All-cause survival in patients with NF1 (screened and unscreened) compared with screened family history clinic patients without NF1 stratified by tumor grade. The follow-up of patients with NF1 starts either at the first breast cancer, or the latter of the first breast cancer and entry to NF1 cohort (delayed entry). Early screening in the non-NF1 group consists of annual mammography at 30-49 years of age and 18-monthly screening at 50-59 years of age.

importantly there were no breast cancer deaths in the Padova NF1 population.

Tumor pathology for the cases included in the delayed entry analysis is presented in Table 3 . The NF1 population had lower rates of in situ disease than the screened non-NF1 group as might be expected without mammographic screening. The invasive cancers were much less likely to be stage 1 in the NF1 cohorts without screening than in the non-NF1 group $(20.5 \%$ vs. $59.3 \% ; P<0.0001)$. There was also a higher rate of HER $2+$ cancers at $24.1 \%$ among patients with NF1 versus $11.1 \%$ among patients without NF1 $(P=0.03)$. Otherwise tumors in the NF1 and non-NF1 groups were well matched for age at diagnosis, grade, and ER status. However, of the NF1 cohorts the more highly ascertained Manchester and Finnish populations had poorer prognosis cancers with higher proportions of HER2+, ER-, and higher stage cancers. Patients with NF1 and ER + breast cancer had better survival after a median follow-up of 6.1 years since breast cancer, range 0.28 to 18.7 (ER+ vs. ER-, HR 2.8 (95\% CI 1.2-6.8), $P=0.022)$. Unscreened grade 3 cancers in women carrying NF1 gene defects had the worst 10-year survival at $44.2 \%$ (95\% CI 29.3-66.9\%). The NF1 group had worse all-cause survival than the non-NF1 group irrespective of tumor grade (Fig. 2d).

\section{DISCUSSION}

The present study has shown that women with NF1 and breast cancer have a high rate of contralateral breast cancer and is in keeping with the increased risk of a first primary breast cancer of 4-11 fold in six cohort studies of women aged $<50$ years of age. ${ }^{7-13}$ This $1.1 \%$ risk annually is about twice the expected risk from women without familial breast cancer, ${ }^{21}$ but less than the $2 \%$ annual risk for a carrier of a pathogenic variant in $B R C A 1 / 2 .{ }^{22}$ As such the risk for a woman with NF1 


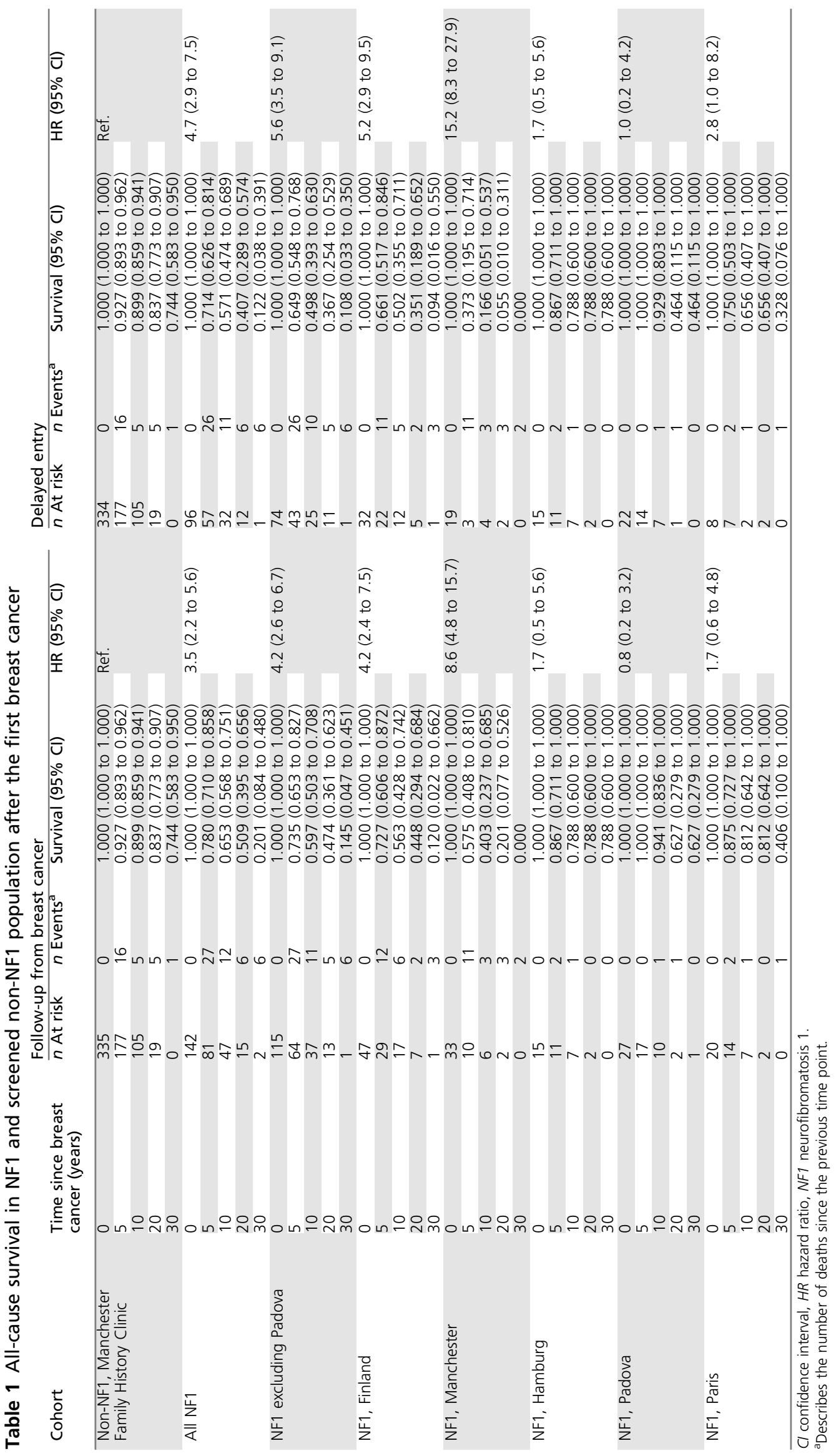




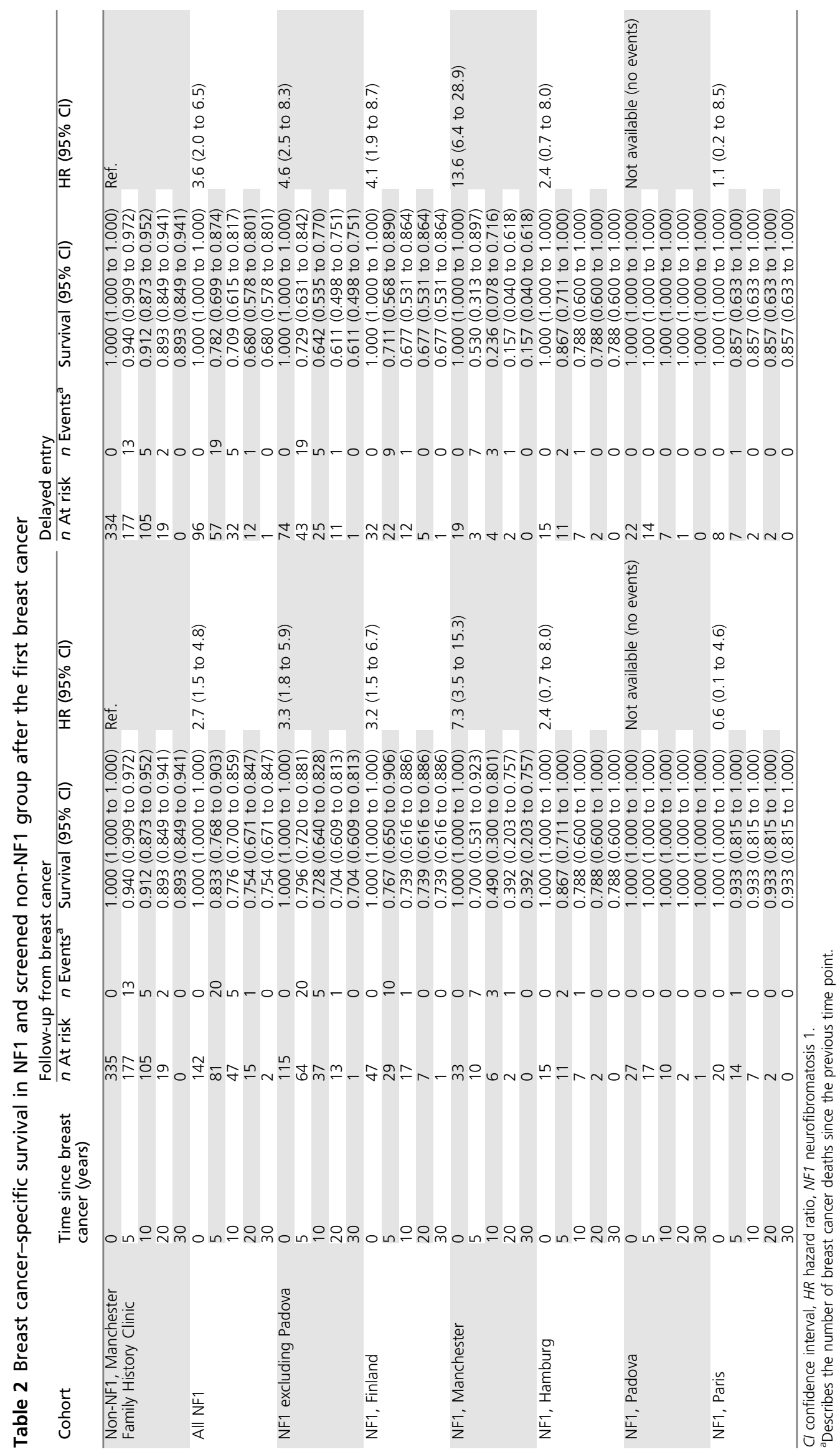




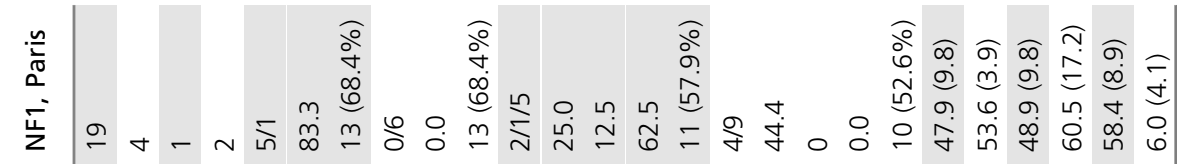

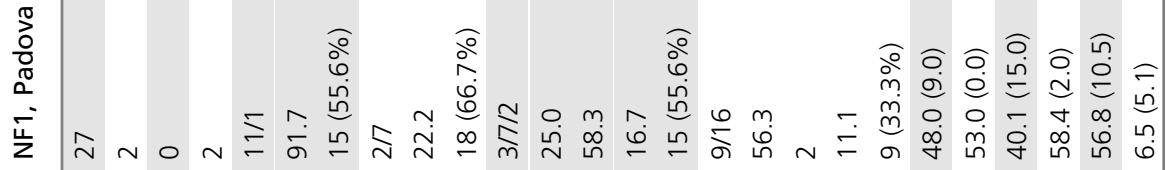

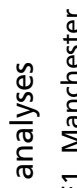

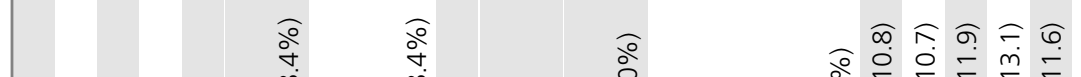

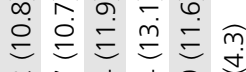

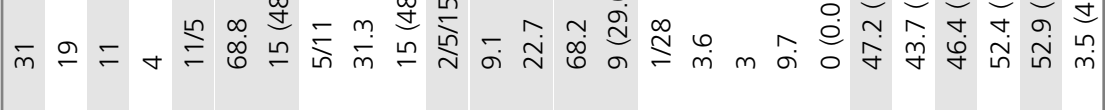

\begin{tabular}{ll}
$\frac{0}{0}$ \\
$\frac{0}{0}$ \\
$\frac{0}{0}$ \\
$\frac{0}{0}$ \\
$\frac{0}{2}$ \\
\hline
\end{tabular}

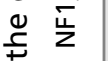

(2)

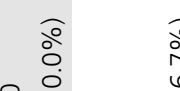

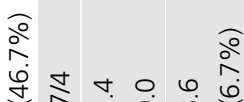

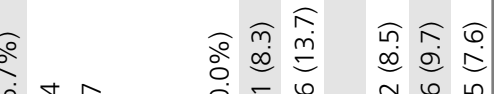

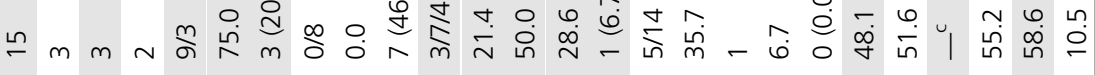

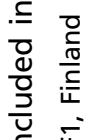

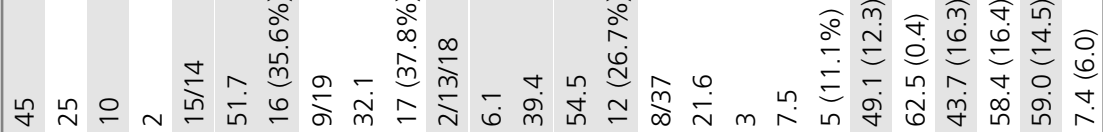

s.

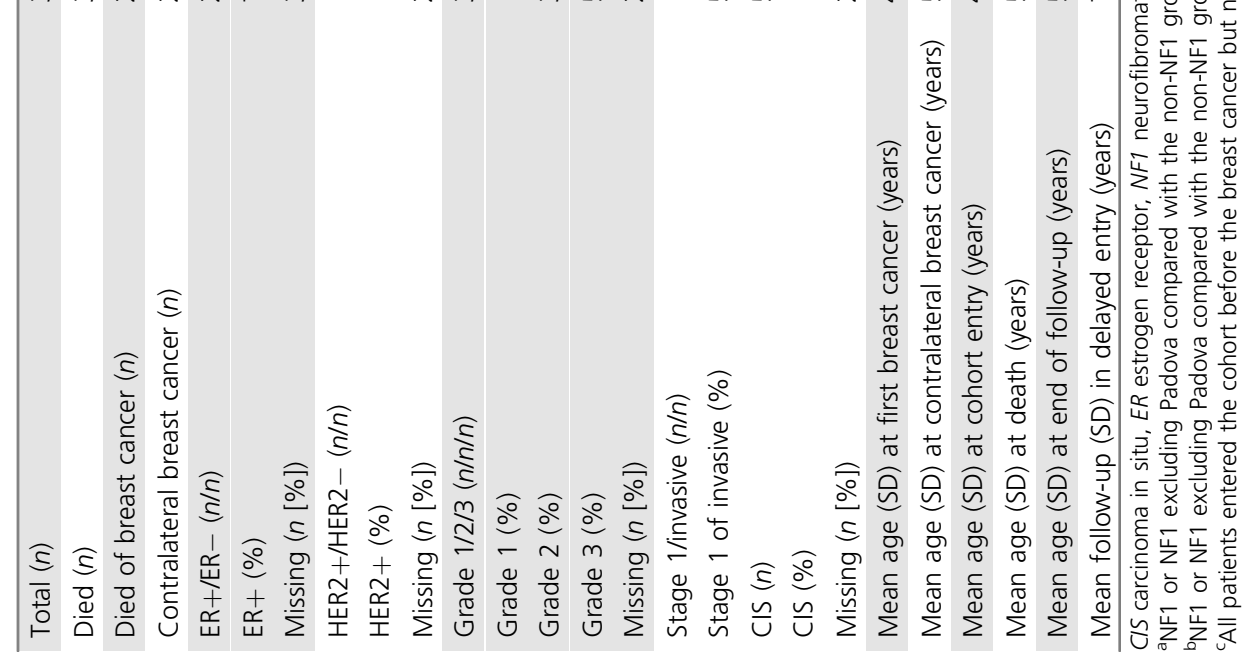


appears intermediate between average risk and $B R C A$-related risk. Indeed it is equivalent to the risk in the non-NF1 cohort, which was predominantly women with non-BRCA familial risk. $^{20}$ The risks would appear sufficient to consider contralateral mastectomy for prevention. $^{22}$ Women with NF1 surviving to 20 years after their first breast cancer had a near $27 \%$ chance of a contralateral breast cancer.

Breast cancer survival in NF1 was very poor especially in the more highly ascertained Manchester and Finnish populations. As expected higher grade and ER- status were associated with poorer survival. Importantly the screened women in Padova had no breast cancer-specific deaths and, where known, had low stage tumors. In addition to differences in screening routines, the five NF1 cohorts differ in terms of their origin: the cohorts from Finland and Manchester are population based whereas the Hamburg, Padova, and Paris cohorts are from specialized clinics. The latter may have patients with better access to health care or greater interest in their health, and the increased surveillance provided in these centers could also affect the prognosis. There are also differences between the participating countries in breast cancer screening and management. For example, some countries initiated general population mammography screening earlier than others.

The effects of screening women at risk of familial breast cancer can be seen by the good survival in the Manchester family history clinic with breast cancer-specific survival of $91 \%$ at 10 years and $89 \%$ at 20 years. ${ }^{20}$ As this population was of a similar age, grade, and ER status as the NF1 group of the current study (Table 3) this reflects what may be possible with more intensive breast screening. The Manchester family history cohort was included to represent an average of a population with mostly moderate risk of breast cancer for benchmarking the survival observed in women with NF1. However, despite group-level characteristics similar to the women with NF1, it should be kept in mind that the non-NF1 population with family history of breast cancer may be relatively heterogeneous in terms of cancer risk and tumor characteristics. Moreover, the different breast cancer predisposing mechanisms in NF1 and in patients with family history of breast cancer may also have different effects on the tumor biology and treatment resistance affecting survival. Screening with annual mammography in those at familial risk aged 40-49 has been shown likely to be beneficial. ${ }^{23}$ As a result National Institute for Health and Care Excellence (NICE) guidance in the UK recommends annual mammography screening aged 40-49 in those with at least moderate risk (10-year risk 3\%+; twofold relative risk). ${ }^{24}$ Guidance in North America would suggest screening even earlier. ${ }^{25}$ A case could be made for magnetic resonance imaging (MRI) screening, which has proven so successful in BRCA1/2 pathogenic variant carriers, ${ }^{26}$ with proven survival benefits. ${ }^{27}$ However, current UK guidelines ${ }^{24}$ would not suggest that women with NF1 would qualify although they would clearly meet the risk criteria in the United States. ${ }^{25,28}$
The long-term survival of women with NF1 post-breast cancer diagnosis is extremely poor with 20-year (delayed entry) survival of only $37 \%$ compared with $84 \%$ in the nonNF1 familial population with very similar ages at diagnosis. Although the non-NF1 population is matched on breast cancer risk and age it does not represent what might happen in a population with no additional screening. However, also the 5-year survival of $64.9 \%$ observed among unscreened patients with NF1 is markedly worse than the 5-year net survival of $79.8-88.5 \%$ reported in the general population in the UK, Finland, Italy, Germany, and France. ${ }^{29}$ The poor long-term survival in women with NF1 and breast cancer may be due to the poor overall life expectancy in $\mathrm{NF} 1^{7,18,30}$ and although breast cancer deaths were relatively rare after 10 years it is possible that breast cancer treatments may have exacerbated the known increased risks of malignancy and cardiovascular disease, especially post-irradiation. ${ }^{7,18,30,31}$

The current study has some limitations. Tumor pathology and receptor status were not available on all women reflecting the over 40-year period of ascertainment. No information was available on whether the tumors were detected in screening or due to symptoms. In the NF1 cohorts without additional screening, $60.0 \%$ of women were too young at cancer diagnosis to participate in the general population screening programs, which suggests that most of these cancers were symptomatic at diagnosis. The non-NF1 population of family history women was in only one country (UK), however this was the country in which patients with NF1 had the poorest breast cancer survival. Given the relative rarity of NF1 the study size is large with 142 women and the contralateral risks and poor survival are likely accurate reflections of the prognosis for women with NF1 and breast cancer.

In conclusion the present multicenter study has shown that women with NF1 have a substantial risk of a contralateral breast cancer and have overall poor survival. Screening in one clinic in Italy suggests that the better prognosis seen in screening those at high familial risk may well substantially improve survival in women with NF1 and breast cancer and we recommend the inclusion of women with NF1 gene defects in national high-risk screening protocols.

\section{ACKNOWLEDGEMENTS}

The authors wish to acknowledge NHS England for their support of the national complex NF1 center. D.G.R.E., E.F.H., and S.J.H. are supported by the all Manchester National Institute for Health Research (NIHR) Biomedical Research Centre (IS-BRC-121520007). R.A.K. is supported by the Finnish Cultural Foundation. S.P. and J.P. are supported by the Cancer Society of Finland and Turku University Hospital.

\section{DISCLOSURE}

The authors declare no conflicts of interest.

Publisher's note Springer Nature remains neutral with regard to jurisdictional claims in published maps and institutional affiliations. 


\section{REFERENCES}

1. Evans DG, Howard E, Giblin C, et al. Birth incidence and prevalence of tumor-prone syndromes: estimates from a UK family genetic register service. Am J Med Genet A. 2010;152A:327-332.

2. Uusitalo $E$, Leppävirta J, Koffert $A$, et al. Incidence and mortality of neurofibromatosis: a total population study in Finland. J Invest Dermatol. 2015;135:904-906.

3. Easton DF, Ponder MA, Huson SM, Ponder BA. An analysis of variation in expression of neurofibromatosis (NF) type 1 (NF1): evidence for modifying genes. Am J Hum Genet. 1993;53:305-313.

4. Abramowicz A, Gos M. Neurofibromin in neurofibromatosis type 1mutations in NF1gene as a cause of disease. Dev Period Med. 2014;18:297-306

5. Evans DG, Bowers N, Burkitt-Wright E, et al. Comprehensive RNA analysis of the NF1 gene in classically affected NF1 meeting NIH criteria has high sensitivity and mutation negative testing is reassuring in isolated cases with pigmentary features only. EBioMedicine. 2016;7:212-220.

6. Evans DGR, Baser ME, McGaughran J, Sharif S, Donnelly B, Moran A. Malignant peripheral nerve sheath tumours in neurofibromatosis 1 . J Med Genet. 2002;39:311-314.

7. Uusitalo $E$, Rantanen $M$, Kallionpää RA, et al. Distinctive cancer associations in patients with neurofibromatosis type 1. J Clin Oncol. 2016;34:1978-1986.

8. Walker L, Tompson D, Easton D, et al. A prospective study of neurofibromatosis type 1 cancer incidence in the UK. Br J Cancer. 2006; 95:233-238.

9. Sharif S, Moran A, Huson SM, et al. Women with neurofibromatosis 1 (NF1) are at a moderately increased risk of developing breast cancer and should be considered for early screening. J Med Genet. 2007;44:481-484.

10. Wang $X$, Levin AM, Smolinski SE, Vigneau FD, Levin NK, Tainsky MA. Breast cancer and other neoplasms in women with neurofibromatosis type 1: a retrospective review of cases in the Detroit metropolitan area. Am J Med Genet A. 2012;158:3061-3064.

11. Seminog OO, Goldacre MJ. Risk of benign tumours of nervous system, and of malignant neoplasms, in people with neurofibromatosis: population-based record-linkage study. Br J Cancer. 2013;108:193-198.

12. Uusitalo $E$, Kallionpää RA, Kurki S, et al. Breast cancer in neurofibromatosis type 1: overrepresentation of unfavourable prognostic factors. Br J Cancer. 2017:116:211-217.

13. Madanikia SA, Bergner $A, Y e X$, Blakeley JO. Increased risk of breast cancer in women with NF1. Am J Med Genet A. 2012:158A:3056-3060.

14. The Cancer Genome Atlas Network. Comprehensive molecular portraits of human breast tumours. Nature. 2012:490:61-70.

15. Suárez-Cabrera C, Quintana RM, Bravo A, et al. Transposon-based analysis reveals RASA1 is involved in triple-negative breast cancer. Cancer Res. 2017:77:1357-1368.

16. Wallace $M D$, Pfefferle $A D$, Shen $L$, et al. Comparative oncogenomics implicates the neurofibromin 1 gene (NF1) as a breast cancer driver. Genetics. 2012;192:385-396.

17. Lammert $M$, Friedman JM, Kluwe $L$, Mautner VF. Prevalence of neurofibromatosis 1 in German children at elementary school enrollment. Arch Dermatol. 2005:141:71-74.

18. Duong TA, Sbidian E, Valeyrie-Allanore L, Vialette C, Ferkal S, Hadj-Rabia $\mathrm{S}$, et al. Mortality associated with neurofibromatosis 1 : a cohort study of 1895 patients in 1980-2006 in France. Orphanet J Rare Dis. 2011;6:18.

19. Trevisson E, Cassina M, Opocher E, et al. Natural history of optic pathway gliomas in a cohort of unselected patients affected by neurofibromatosis 1. J Neurooncol. 2017;134:279-287.

20. Basu NN, Ross GL, Evans DG, Barr L. The Manchester guidelines for contralateral risk-reducing mastectomy. World J Surg Oncol. 2015; 13:237.
21. Basu NN, Ingham S, Hodson J, et al. Risk of contralateral breast cancer in BRCA1 and BRCA2 mutation carriers: a 30-year semi-prospective analysis. Fam Cancer. 2015;14:531-538.

22. Maurice A, Evans DG, Affen J, Greenhalgh R, Duffy SW, Howell A. Surveillance of women at increased risk of breast cancer using mammography and clinical breast examination: further evidence of benefit. Int J Cancer. 2012;131:417-425.

23. FH01 collaborative teams. Mammographic surveillance in women younger than 50 years who have a family history of breast cancer: tumour characteristics and projected effect on mortality in the prospective, single-arm FH01 study. Lancet Oncol. 2010;11:1127-1134.

24. National Institute for Health and Care Excellence (NICE). Familial breast cancer: classification, care and managing breast cancer and related risks in people with a family history of breast cancer. https://www.nice.org.uk/ guidance/cg164/chapter/recommendations\#summary-ofrecommendations-on-surveillance-for-women-with-no-personal-historyof-breast-cancer. Accessed 3 October 2018.

25. National Comprehensive Cancer Network (NCCN). NCCN guidelines for detection, prevention, \& risk reduction: Genetic/Familial High-Risk Assessment: Breast and Ovarian. https://www.nccn.org/professionals/ physician_gls/f_guidelines.asp\#genetics_screening. Accessed 23 September 2018.

26. Leach $M O$, Boggis $C R$, Dixon $A K$, et al. Screening with magnetic resonance imaging and mammography of a UK population at high familial risk of breast cancer: a prospective multicentre cohort study (MARIBS). Lancet. 2005;365:1769-1778.

27. Evans DG, Kesavan N, Lim $Y$, et al. MRI breast screening in high-risk women: cancer detection and survival analysis. Breast Cancer Res Treat. 2014;145:663-672.

28. Daly MB, Pilarski R, Berry M, et al. NCCN Guidelines insights: genetid familial high-risk assessment: breast and ovarian, version 2.2017. J Natl Compr Canc Netw. 2017:15:9-20.

29. Allemani $C$, Matsuda T, Di Carlo V, et al. Global surveillance of trends in cancer survival 2000-14 (CONCORD-3): analysis of individual records for 37513025 patients diagnosed with one of 18 cancers from 322 population-based registries in 71 countries. Lancet. 2018:391:1023-1075.

30. Evans DG, O'Hara C, Wilding A, et al. Mortality in neurofibromatosis 1 : in North West England: an assessment of actuarial survival in a region of the UK since 1989. Eur J Hum Genet. 2011:19:1187-1191.

31. Sharif S, Ferner R, Birch JM, Gillespie JE, Gattamaneni HR, Baser ME, et al. Second primary tumors in neurofibromatosis 1 patients treated for optic glioma: substantial risks after radiotherapy. J Clin Oncol. 2006:24:2570-2575.

Open Access This article is licensed under a Creative Commons ccc
License, which permits any non-commercial use, sharing, distribution and reproduction in any medium or format, as long as you give appropriate credit to the original author(s) and the source, and provide a link to the Creative Commons license. You do not have permission under this license to share adapted material derived from this article or parts of it. The images or other third party material in this article are included in the article's Creative Commons license, unless indicated otherwise in a credit line to the material. If material is not included in the article's Creative Commons license and your intended use is not permitted by statutory regulation or exceeds the permitted use, you will need to obtain permission directly from the copyright holder. To view a copy of this license, visit http://creativecommons.org/licenses/by-nc-nd/4.0/.

(c) The Author(s) 2019 